\title{
Gelatin Behaviour in Dilute Aqueous Solution: Designing a Nanoparticulate Formulation
}

\author{
CLAUDE A. FARRUGIA AND MICHAEL J. GROVES
}

\author{
Institute for Tuberculosis Research, College of Pharmacy, University of Illinois at Chicago, Chicago, \\ IL 60607, USA
}

\begin{abstract}
Although it has been claimed that nanoparticles can be produced from gelatin, a naturally occurring polypeptide, the commercial conversion of animal collagen to gelatin results in a heterogeneous product with a wide molecular-weight range. This is probably responsible for the widely observed variation in the experimental conditions required for nanoparticle formation.

In this study, $0.2 \% \mathrm{w} / \mathrm{v}$ aqueous B225 gelatin solutions were incubated under various conditions of time, temperature, $\mathrm{pH}$ and ethanol concentration and characterized by both size-exclusion high-performance liquid chromatography (HPLC) and dynamic light scattering. Gelatin was shown to be denatured when the temperature was increased to $37^{\circ} \mathrm{C}$ (approx.) and the rate of renaturation was optimized over the temperature range $7-20^{\circ} \mathrm{C}$ at $\mathrm{pH} 5.0$, equivalent to the isoelectric point (IEP). The molecular-weight profile remained unchanged at $37^{\circ} \mathrm{C}$ (approx.) in the $\mathrm{pH}$ range 5-7. When the gelatin solutions were mixed with ethanol, higher-molecular-weight fractions (microgel, $\delta$ and $\zeta$ fractions, all with molecular weights $>700 \mathrm{kDa}$ ) precipitated at ethanol concentrations lower than those required to precipitate the lower molecular weight material $(<700 \mathrm{kDa})$, with maximum precipitation occurring close to the isoelectric point $(\mathrm{pH} 5 \cdot 0)$.

The molecular weight profile of gelatin in solution is evidently critically affected in a time-dependent manner by both $\mathrm{pH}$ and temperature. These two factors influence the noncovalent interactions responsible for the molecular structure of gelatin. The molecular weight profiles, in turn, affect the phase behaviour of gelatin in hydroalcoholic solutions. Systematically investigating the effect of time, temperature, $\mathrm{pH}$ and ethanol concentration on the molecular-weight-distribution profile of a gelatin solution enabled a robust method to be developed for the preparation of colloidal dispersions of non-aggregated gelatin nanoparticles $220-250 \mathrm{~nm}$ in diameter. This contrasts with the multiparticulate aggregates produced by earlier literature methods.
\end{abstract}

Nanoparticles (dispersed particulate systems with mean diameters below $1 \mu \mathrm{m}$ ) have promise as target-oriented drug-delivery systems and can be prepared from a variety of natural or synthetic macromolecules. Although the use of gelatin as a base for nanoparticles has been well-documented (Marty et al 1978; El-Samaligy \& Rohdewald 1982, 1983) it has been reported that the success of the technique requires experienced professional

Correspondence: M. J. Groves, Institute for Tuberculosis Research, College of Pharmacy, University of Illinois at Chicago (M/C 964), Room 2014 SEL, 950 South Halsted Street, Chicago, IL 60607, USA. operators and that different lots of nominally the same gelatin require different experimental conditions for nanoparticle formation (Oppenheim 1986).

The commercial conversion of animal collagen to gelatin involves acidic or basic cleavage of the intermolecular and intramolecular covalent bonds which stabilize collagen and render it insoluble (Courts 1954). This generates soluble tropocollagen molecules which are further denatured by breakage of hydrogen-bonds and disruption of the triplehelix structure (Flory \& Weaver 1960; Steven \& Tristram 1962). Although these two processes 
would be expected to yield a preparation of $\alpha$ chains with an average molecular weight of approximately $95 \mathrm{kDa}$, the heterogeneous nature of the reactions involved results in some intramolecular covalent links remaining intact whereas other peptide bonds, particularly those involving glycine, are more labile (Johns \& Courts 1977). This denaturation has been shown to be partially reversible; Von Hippel \& Harrington (1959) and Flory \& Weaver (1960) observed limited reformation of gelatin to the collagen structure in dilute solution. The net result of these diverse processes is a final product containing components with a wide range of molecular weights. Previous studies identified a several fractions present in aqueous gelatin solutions. The sub- $\alpha$ fraction $(50-80 \mathrm{kDa})$ consists of hydrolysis fragments of the $\alpha$ fraction (80$125 \mathrm{kDa}$ ) (Lorry \& Vedrines 1985; Steckert et al 1992 ), itself corresponding to the $\alpha$-chains derived from the tropocollagen molecule (Piez et al 1960; Veis et al 1962). These $\alpha$-chains associate with each other to form the $\beta$ fraction $(125-230 \mathrm{kDa})$ and the $\gamma$ fraction $(230-340 \mathrm{kDa})$ (Lorry \& Vedrines 1985). These also correspond to the $\beta$ chains and $\gamma$-chains derived from tropocollagen (Piez et al 1960, 1961; Veis et al 1962). The $\delta$ fraction, with a molecular weight of $1400 \mathrm{kDa}$ (approx.) corresponds to a tetramer of the $\gamma$ component with a large amount of cross-linking (Veis et al 1962).

This molecular weight heterogeneity present in a solution of gelatin is evidently responsible for the observed variation in the experimental conditions required for gelatin nanoparticle formation, particularly when the technique involves separation of a coacervate phase by desolvation (Marty et al 1978). Elysée-Collen \& Lencki (1996) showed that the phase behaviour of gelatin was extremely complex and influenced by the temperature of the medium. This reflected the initial heterogeneity of the molecular-weight-distribution of the gelatin sample and might explain the observed sensitivity to the experimental conditions in the production of gelatin nanoparticles by addition of solvents.

In this paper we describe the time-dependent effect of temperature and $\mathrm{pH}$, and the effect of the presence of a desolvating agent, on the molecularweight characteristics of a gelatin solution, as measured by size-exclusion chromatography.

\section{Materials and Methods}

\section{Materials}

All chemicals were of analytical reagent-grade quality. Gelatin, from bovine skin, lime-cured
(Type B), with bloom strength of 225, and phenylmethylsulphonyl fluoride were from Sigma (St Louis, MO). Sodium chloride, sodium phosphate monobasic monohydrate, sodium phosphate dibasic anhydrous, citric acid, ethanol, sodium metabisulphite, glutaraldehyde and Tween 20 were from Fisher Scientific (Itasca, IL).

High-performance liquid chromatography (HPLC) The HPLC system (Waters, Milford, MA) comprised a model 501 pump and a model 486 tuneable absorbance detector operated at $205 \mathrm{~nm}$. Millennium 2010 Chromatography Manager software was used to control the HPLC system and perform data acquisition. The mobile phase was phosphate-buffered saline (PBS). Solutions were freshly prepared in degassed, double-distilled water and vacuumfiltered through a $0 \cdot 2-\mu \mathrm{m}$ Supor-200 membrane filter (Fisher Scientific). The mobile phase was warmed to $30^{\circ} \mathrm{C}$ before use and maintained at that temperature throughout the procedure. The HPLC apparatus was calibrated by use of a gel-filtration chromatography standard from Bio-Rad Laboratories (Hercules, CA) containing thyroglobulin $(670 \mathrm{kDa})$, bovine $\gamma$-globulin $(158 \mathrm{kDa})$, chicken ovalbumin $(44 \mathrm{kDa})$, equine myoglobin $(17 \mathrm{kDa})$ and vitamin $\mathrm{B}_{12}(1.35 \mathrm{kDa})$.

\section{Gelatin HPLC characterization}

Gelatin solutions $(0.2 \% \mathrm{w} / \mathrm{v})$ were prepared in degassed, double-distilled water, which had been vacuum-filtered through a $0 \cdot 2-\mu \mathrm{m}$ Supor-200 membrane filter, by heating to $40^{\circ} \mathrm{C}$ with stirring for $20 \mathrm{~min}$ then cooling the unstirred solution to $20^{\circ} \mathrm{C}$ for $90 \mathrm{~min}$. The gelatin solution was passed through an Ultrahydrogel Linear size-exclusion column (Waters) at $0.3 \mathrm{~mL} \mathrm{~min}^{-1}$.

\section{Incubation experiments}

Gelatin solutions were prepared, as described above, in $0.1 \mathrm{M} \mathrm{pH} 7$ or $\mathrm{pH} 5$ phosphate buffer or in $0.05 \mathrm{M} \mathrm{pH} 3$ citric acid-sodium citrate buffer (Perrin \& Dempsey 1974). Phenylmethylsulphonyl fluoride $(1 \mathrm{mM})$ was added as a protease inhibitor and the $\mathrm{pH}$ of the solution was checked by use of an Accumet model $925 \mathrm{pH} /$ ion meter (Fisher Scientific). Solutions were incubated at 7, 20, 37, 56 or $80^{\circ} \mathrm{C}$ and samples were removed at regular intervals over a 2-day period and filtered through a $0 \cdot 22-$ $\mu \mathrm{m}$ Durapore membrane filter (Millipore). These samples were analyzed by size-exclusion HPLC as described above. The chromatograms generated were subdivided into molecular-weight ranges and 
Table 1. Molecular-weight classes for analysis of gelatin HPLC chromatograms, and their percentage composition in $0.2 \% \mathrm{w} / \mathrm{v}$ B225 gelatin solution after $0 \mathrm{~h}$ incubation.

\begin{tabular}{lccc}
\hline $\begin{array}{l}\text { Molecular } \\
\text { weight class }\end{array}$ & $\begin{array}{c}\text { Molecular } \\
\text { weight } \\
\text { range (kDa) }\end{array}$ & $\begin{array}{c}\text { Approximate } \\
\text { number of } \\
\alpha \text { chains }\end{array}$ & $\begin{array}{c}\% \text { Composition } \\
(\% \text { mean } \pm \text { s.d.) }\end{array}$ \\
\hline $\begin{array}{l}\text { Low molecular- } \\
\quad \text { weight fraction }\end{array}$ & $<50$ & - & $13 \cdot 5 \pm 0 \cdot 7$ \\
$\begin{array}{l}\text { Sub-alpha } \\
\text { Alpha }(\alpha)\end{array}$ & $50-80$ & - & $6 \cdot 1 \pm 0 \cdot 3$ \\
Beta $(\beta)$ & $80-125$ & 1 & $8 \cdot 1 \pm 0 \cdot 4$ \\
Gamma $(\gamma)$ & $125-225$ & 2 & $14 \cdot 1 \pm 0 \cdot 5$ \\
Epsilon $(\varepsilon)$ & $225-340$ & 3 & $10.5 \pm 0 \cdot 3$ \\
Zeta $(\zeta)$ & $740-700$ & $6(2 \gamma)$ & $16 \cdot 2 \pm 0 \cdot 3$ \\
Delta $(\delta)$ & $1000-1000$ & $9(3 \gamma)$ & $7 \cdot 3 \pm 0 \cdot 1$ \\
Microgel & $>1800$ & $12(4 \gamma)$ & $10.6 \pm 0 \cdot 3$ \\
& & $>12$ & $13.4 \pm 1 \cdot 7$ \\
\hline
\end{tabular}

the percentage composition of each class was calculated from the area under the curve (AUC). The percentage compositions (Table 1) were then subjected to kinetic analysis with the software program Prism (GraphPad Software).

\section{Desolvation experiments}

Unbuffered gelatin solutions were prepared by heating aqueous suspensions of undissolved gelatin to $40^{\circ} \mathrm{C}$ with stirring for $20 \mathrm{~min}$ and the $\mathrm{pH}$ was adjusted to 3,5 , or 7 with dilute hydrochloric acid or dilute sodium hydroxide. The gelatin solutions were then incubated at 20,37 or $56^{\circ} \mathrm{C}$ for $1.5 \mathrm{~h}$ and mixed with hydroalcoholic solutions that had been similarly incubated. The final solutions contained $0.2 \% \mathrm{w} / \mathrm{w}$ gelatin and increasing amounts of ethanol $(40-75 \% \mathrm{w} / \mathrm{w}$, in $5 \%$ increments). The three-component mixtures were incubated at the same temperature for a further $20 \mathrm{~min}$. Samples from these solutions were filtered and analyzed by size-exclusion HPLC as described above. The turbidity of the solutions was also monitored, by lightscattering, by means of a Malvern Zetasizer 3 (Malvern Instruments, Southborough, MA) and by transmittance, by use of a Beckman DU-65 UV-Vis spectrophotometer operated at $600 \mathrm{~nm}$ (Beckman Instruments, Fullerton, CA).

\section{Preparation of gelatin nanoparticles}

Gelatin nanoparticles were prepared from a $1 \%$ $\mathrm{w} / \mathrm{w}$ aqueous gelatin solution by adjusting the $\mathrm{pH}$ to between 5.5 and 8.5 with dilute sodium hydroxide and incubating at $37^{\circ} \mathrm{C}$ for $1.5 \mathrm{~h}$. Hydroalcoholic solutions, similarly incubated, were then added to the gelatin solution to give final mixtures containing $0.2 \% \mathrm{w} / \mathrm{w}$ gelatin and between 62.5 and $75 \% \mathrm{w} / \mathrm{w}$ ethanol. The mixtures were incubated at $37^{\circ} \mathrm{C}$ for a further $20 \mathrm{~min}$ and diluted $1: 30$ by weight, with stirring, with hydroalcoholic solutions of similar composition and at the same temperature, but containing $1 \% \mathrm{w} / \mathrm{w}$ glutaraldehyde. The particles were reacted for $2 \mathrm{~h}$ and excess glutaraldehyde neutralized by addition of $3 \% \mathrm{w} / \mathrm{v}$ sodium metabisulphite solution with stirring for $10 \mathrm{~min}$. Separation of the particles was performed by removing ethanol and residual low-molecularweight gelatin fractions by ultrafiltration through an Amicon XM300 membrane (Millipore); a 1\% w/v aqueous solution of Tween 20 was used as the washing agent. The nanoparticles were then sized by means of a Malvern Zetasizer 3 and a Jeol JSM$35 \mathrm{C}$ scanning electron microscope. For scanning electron microscopy, dispersions of nanoparticles were applied sparingly to $0 \cdot 2-\mu \mathrm{m}$ Supor-200 membrane filters. The membranes with nanoparticles were left to dry for $24 \mathrm{~h}$ before observation and photography. The particles were frozen at $-70^{\circ} \mathrm{C}$ and lyophilized before storage at room temperature for two weeks, when they were again reconstituted and sized by use of the Zetasizer.

\section{Results and Discussion}

\section{HPLC characterization of the gelatin}

The chromatograms obtained by size-exclusion HPLC could be subdivided into ranges, as defined in Table 1. On the basis of the gelatin chromatograms it was necessary to define two additional molecular-weight ranges to classify various highermolecular-weight components into multimers of the previously observed $\gamma$ component. These were the $\varepsilon$ fraction, with a molecular-weight range of $340-700 \mathrm{kDa}$, corresponding to a dimer of the $\gamma$ component, and the $\zeta$ fraction, with a molecularweight range of 700-1000 kDa, effectively a trimer of the $\gamma$ component. These two fractions represent consecutive steps of polymerization of the $\gamma$ chains into their tetramer fraction, the $\delta$ component (Veis et al 1962), before further polymerization into components of even higher molecular weight $(>1800 \mathrm{kDa})$, collectively designated the microgel fraction.

\section{Incubation experiments}

The helical tropocollagen structure characteristic of higher-molecular-weight fractions of gelatin $(\gamma$ fraction and its polymers) can be denatured either by heating (Flory \& Weaver 1960) or by addition of substances that disrupt hydrogen-bonds at room temperature (Steven \& Tristram 1962). Thus 
Table 2. Percentage content of microgel fraction after $24 \mathrm{~h}$.

\begin{tabular}{lrrr}
\hline Temperature $\left({ }^{\circ} \mathrm{C}\right)$ & \multicolumn{1}{c}{$\mathrm{pH} 3$} & $\mathrm{pH} 5$ & \multicolumn{1}{c}{$\mathrm{pH} 7$} \\
\hline 7 & $14 \cdot 9 \pm 0 \cdot 9$ & $23 \cdot 2 \pm 1 \cdot 1$ & $25 \cdot 8 \pm 0 \cdot 8$ \\
20 & $12 \cdot 8 \pm 0 \cdot 3$ & $29 \cdot 3 \pm 0 \cdot 6$ & $23 \cdot 3 \pm 0 \cdot 2$ \\
37 & $4 \cdot 6 \pm 0 \cdot 3$ & $7 \cdot 1 \pm 1 \cdot 2$ & $8 \cdot 1 \pm 1 \cdot 2$ \\
56 & $0 \cdot 0 \pm 0 \cdot 0$ & $3 \cdot 2 \pm 0 \cdot 6$ & $2 \cdot 0 \pm 0 \cdot 8$ \\
80 & $0 \cdot 0 \pm 0 \cdot 0$ & $0 \cdot 6 \pm 0 \cdot 4$ & $0 \cdot 2 \pm 0 \cdot 1$
\end{tabular}

Values are mean \pm s.e.m. of two readings. The percentage content at time $=0 \mathrm{~h}$ was $13.4 \pm 1.7 \%$.

denaturation of the higher-molecular-weight fractions, particularly the microgel fraction, was observed for gelatin solutions incubated at $80^{\circ} \mathrm{C}$ and $56^{\circ} \mathrm{C}$ (Table 2 and Figure 1). At $37^{\circ} \mathrm{C}$ solutions buffered at $\mathrm{pH} 5$ and $\mathrm{pH} 7$ suffered almost no change in composition whereas mild denaturation was observed for that buffered at $\mathrm{pH} 3$ (Figures 1 and 2 ). At $20^{\circ} \mathrm{C}$ and $7^{\circ} \mathrm{C}$ renaturation of the highermolecular-weight fractions was observed; this culminated in increases in the percentage composition of the microgel fraction (Table 2). The greatest rate of renaturation was observed for solutions buffered at $\mathrm{pH} 5$ (Figure 1). This is close to the isoelectric point (IEP) of lime-processed gelatin, generally believed to fall within the range 4.8-5.2 (Jones 1987). At this $\mathrm{pH}$ the lack of net charge on the individual polypeptide units reduced the extent of intermolecular repulsion, favouring the formation of non-covalent interactions.

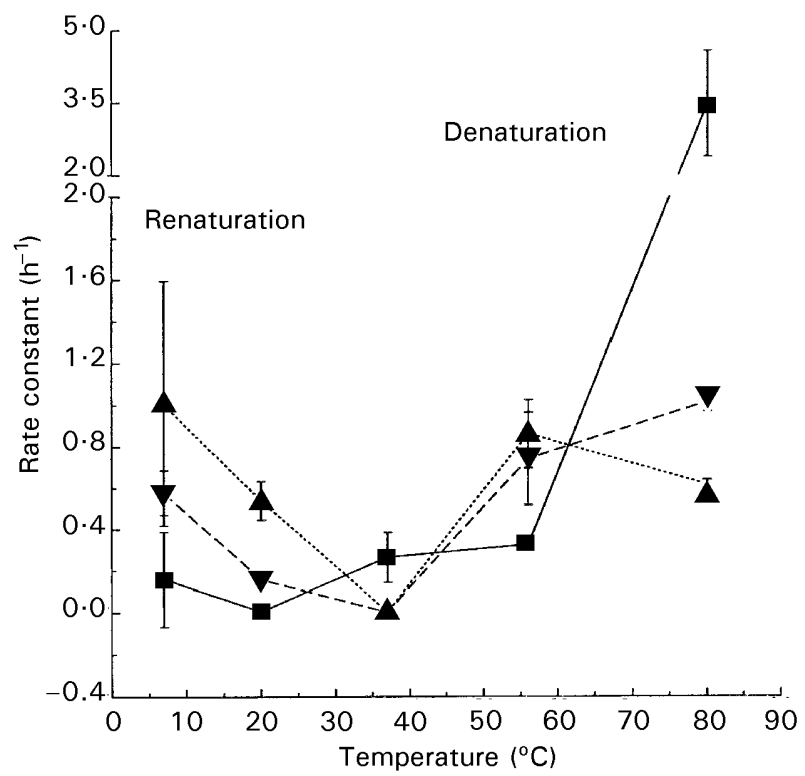

Figure 1. Denaturation and renaturation rate constants $\left(\lambda_{1}\right)$ for the microgel fraction at $\mathrm{pH} 3(\boldsymbol{\square}), \mathrm{pH} 5(\boldsymbol{\Delta})$ and $\mathrm{pH} 7(\boldsymbol{\nabla})$, calculated according to the general equation $\mathrm{B}=$ $\mathrm{P}_{1} \mathrm{e}^{-\lambda 1 \mathrm{t}}+\mathrm{P}_{2} \mathrm{e}^{-\lambda 2 \mathrm{t}}$ (where $\mathrm{B}$ is the concentration of microgel, $\mathrm{P}_{1}$ and $\mathrm{P}_{2}$ are constants determined by the rate constants and starting concentrations, and $\lambda_{1}$ and $\lambda_{2}$ are constants determined by the rate constants) (Cantor \& Schimmel 1980). Values are mean \pm s.e.m. $(\mathrm{df}=6)$.
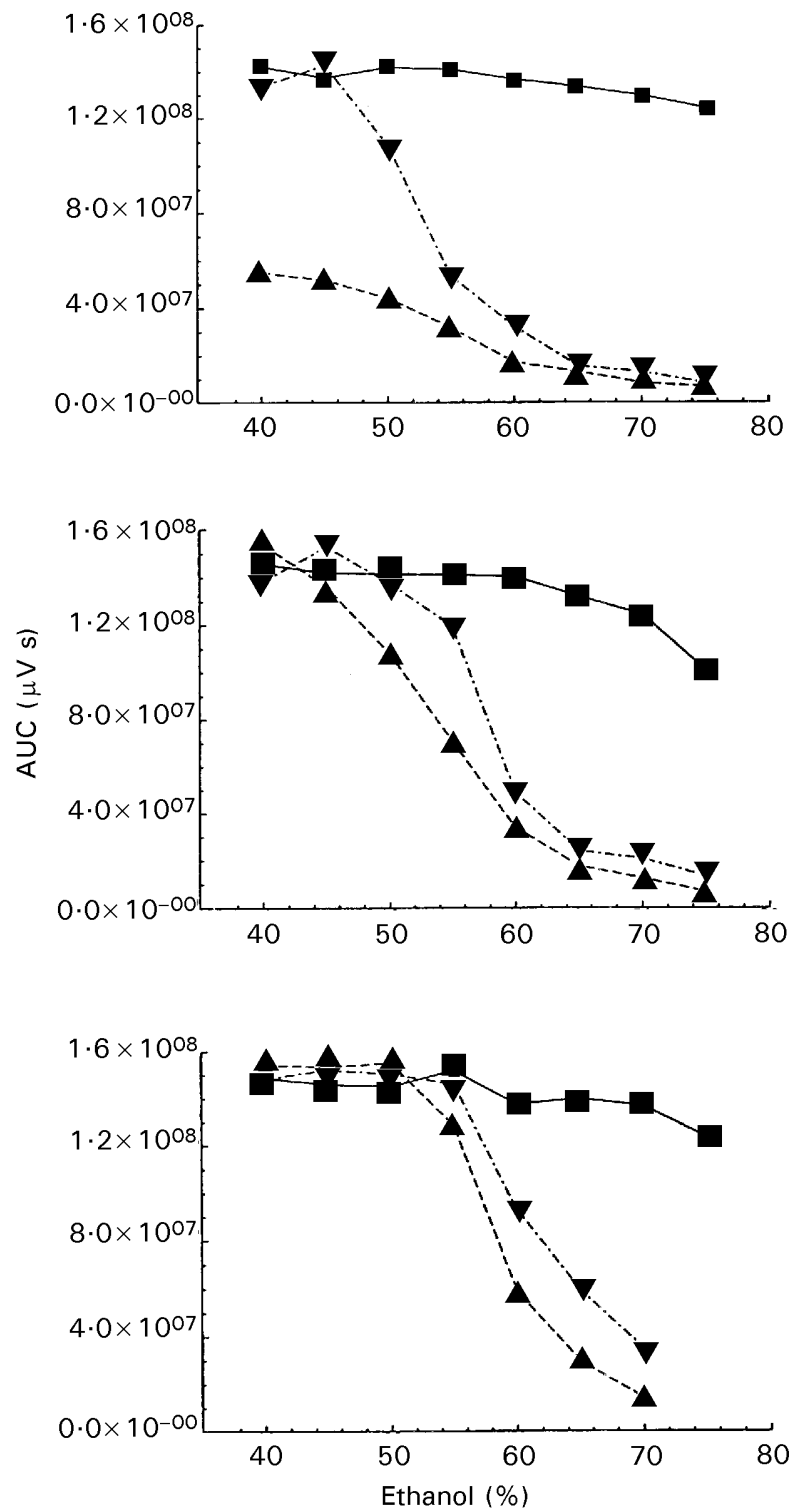

Figure 2. Change in amount of gelatin left in solution at $\mathrm{pH} 3$ $(\boldsymbol{\square}), \mathrm{pH} 5(\boldsymbol{\Delta})$ and $\mathrm{pH} 7(\boldsymbol{\nabla})$, as measured by total AUC, with increase in percentage $\mathrm{w} / \mathrm{w}$ ethanol for gelatin HPLC chromatograms at A. $20^{\circ} \mathrm{C}$, B. $37^{\circ} \mathrm{C}$, and C. $56^{\circ} \mathrm{C}$.

\section{Desolvation experiments}

The folding of proteins into their characteristic three-dimensional shape is determined primarily by non-covalent interactions, which, with the organization of water molecules around the non-polar solute molecules, are largely responsible for the behaviour of proteins in aqueous solution (Privalov $\&$ Gill 1988). Consequently, when a solvent of different polarity and hydrogen-bond-forming capacity such as ethanol replaces some or all of the water molecules, the solvent-solute interactions that determine the solubility of the protein will be significantly affected (Kreuter 1994). At all temperatures gelatin solutions adjusted to $\mathrm{pH} 5$ and 7 became more turbid with increasing ethanol con- 
centration. However, solutions adjusted to $\mathrm{pH} 3$ were insensitive to the desolvating effect, with no increase in turbidity occurring when ethanol concentrations were below $70 \% \mathrm{w} / \mathrm{w}$. This effect was probably related to the more intense net charge present on the gelatin molecules at $\mathrm{pH} 3$, resulting in intermolecular repulsion and reduced aggregation.

The total area under the curve (AUC) for the HPLC chromatograms in these experiments was taken to represent the amount of gelatin left in solution after aggregation by ethanol (Figure 2). The trends in total AUC with increasing ethanol concentrations were comparable with the turbidity readings. Decreases in total AUC and hence in the amount of gelatin left in solution were observed for gelatin solutions adjusted to $\mathrm{pH} 5$ and $\mathrm{pH} 7$, whereas very little decrease in AUC with increasing ethanol concentration was measured for those adjusted to $\mathrm{pH} 3$. The greatest decrease in dissolved gelatin with increasing ethanol concentration was observed for gelatin solutions adjusted to $\mathrm{pH} 5$. The proximity of this $\mathrm{pH}$ to the IEP of the gelatin evidently resulted in a reduced net charge on the gelatin molecules, thereby facilitating aggregation and precipitation.

Dividing the HPLC chromatograms into their component molecular-weight classes enabled determination of the molecular weight fractions that precipitated from the solution as the ethanol content of the mixture increased. The highermolecular-weight fractions (microgel, $\delta$, and $\zeta$ fractions, $>700 \mathrm{kDa}$ ) were the first to come out of solution, followed by the intermediate molecularweight fractions $(\varepsilon, \gamma$ and $\beta$ fractions, 125$700 \mathrm{kDa})$ and finally the low-molecular-weight fractions $(\alpha$, sub- $\alpha$ and LMW fractions, $<125 \mathrm{kDa}$ ), Figure 3. This explained the increasing amount of ethanol required to achieve similar reductions in the amount of dissolved gelatin at different temperatures, as shown in the AUCethanol curves (Figure 2). Gelatin solutions incubated at $20^{\circ} \mathrm{C}$ were renatured towards the highermolecular-weight fractions whereas solutions incubated at $56^{\circ} \mathrm{C}$ suffered mild denaturation; little change in molecular-weight distributions was observed for solutions incubated at $37^{\circ} \mathrm{C}$ (Figures 1 and 2).

\section{Optimization of gelatin nanoparticle production}

We initially prepared gelatin nanoparticles according to the method described by Marty et al (1978), using AUC-percentage ethanol profiles to determine the position of the desolvation region, defined

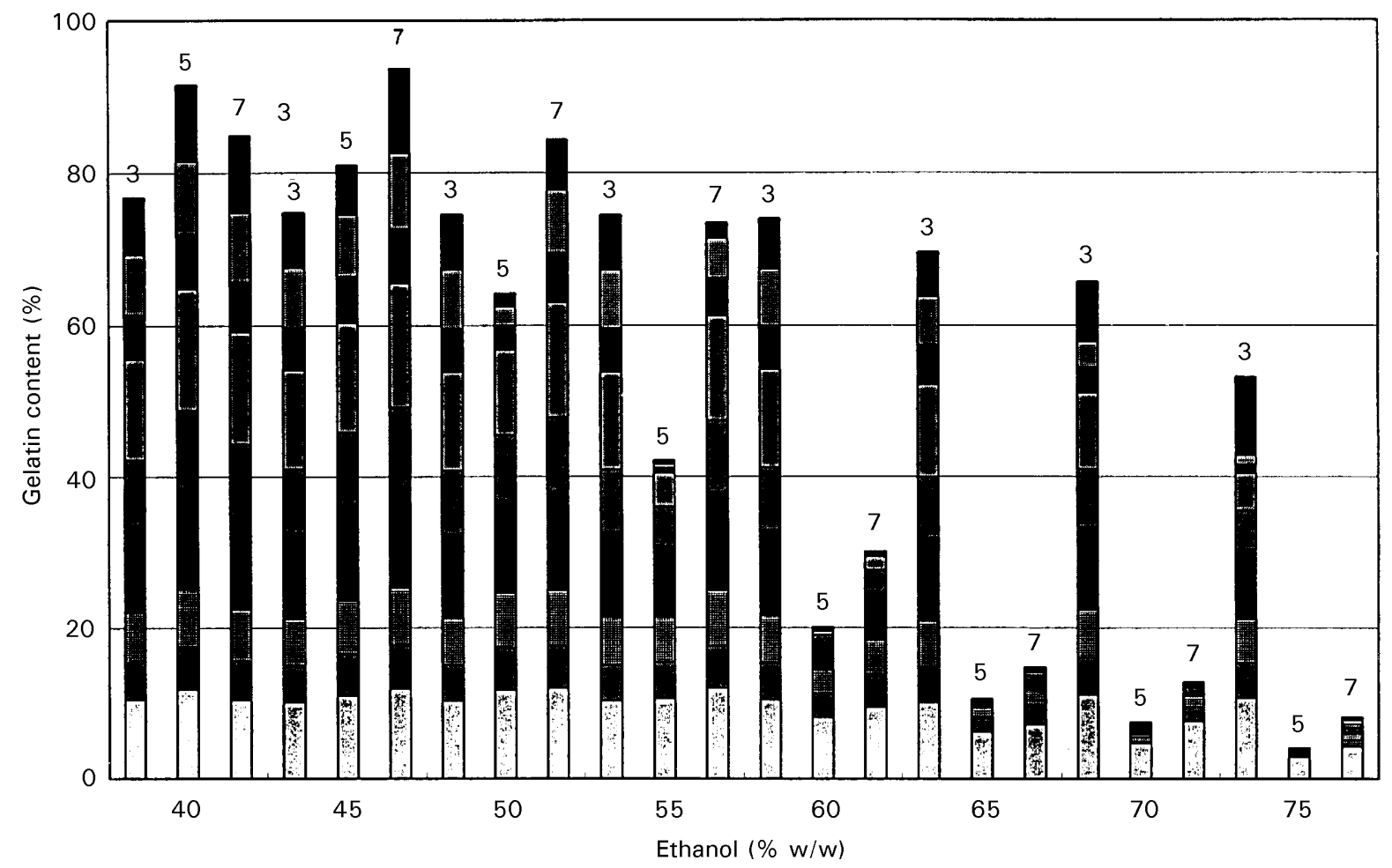

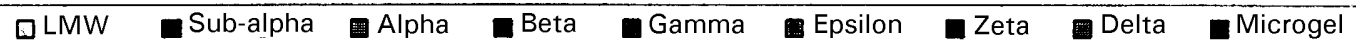

Figure 3. Effect of ethanol on the percentage compositions of the molecular-weight classes at $37^{\circ} \mathrm{C}$. Values are expressed as a percentage of the AUC (which represents the amount of gelatin in solution) for $0 \%$ ethanol. 

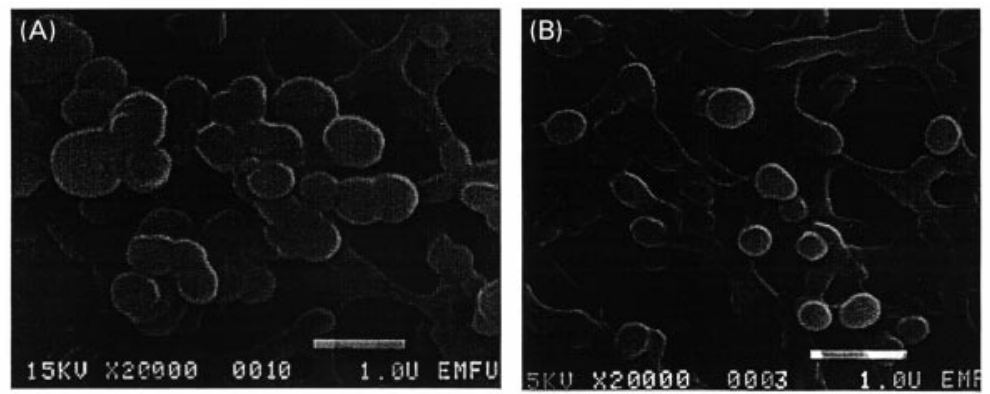

Figure 4. Scanning electron micrographs of nanoparticles on a filter-membrane support, as obtained by the method of Marty et al (1978) (A) and the optimized method (B).

Table 3. Mean size and polydispersity (photon correlation spectroscopy) of gelatin nanoparticle aggregates prepared according to the method of Marty et al (1978).

\begin{tabular}{lcc}
\hline Experimental conditions & Mean Size (nm) & Polydispersity \\
\hline $55 \%$ ethanol, pH7 & Aggregate & - \\
$50 \%$ ethanol, pH 7 & $1082 \cdot 1$ & $0 \cdot 20$ \\
$45 \%$ ethanol, pH 7 & $500 \cdot 6$ & $0 \cdot 21$ \\
$45 \%$ ethanol, pH 5 & $923 \cdot 0$ & 0.47 \\
$40 \%$ ethanol, pH 5 & $465 \cdot 8$ & 0.48 \\
\hline
\end{tabular}

by these authors as the conditions under which the macromolecule was partially desolvated. However, slight differences in experimental conditions resulted in variable size and polydispersity for the nanoparticles produced. Thus, it was noted that if even a slight excess of dehydrating agent such as ethanol was employed, mass aggregation and precipitation of the particles occurred (Table 3). Moreover, for gelatin solutions adjusted to $\mathrm{pH} 5$ the size distributions, as represented by polydispersity readings obtained by photon-correlation spectroscopy, were unacceptably wide. Examination of the particles by scanning electron microscopy confirmed that the differences in measured size were because of the aggregation of primary nanoparticles (Figure 4A).

The higher-molecular-weight fractions (microgel, $\delta$ and $\zeta,>700 \mathrm{kDa}$ ) seem to play a key role in the formation of gelatin nanoparticles by this technique. The desolvation region defined by Marty et al (1978) coincided with the appearance of a decrease in the amount of gelatin left in solution in the AUC-percentage ethanol profiles (Figure 2). The fractions responsible for this decrease belong to the higher-molecular-weight classes $(>700$ $\mathrm{kDa}$ ). Consequently, these results were used to define the criteria necessary for optimization of the experimental conditions. Slight changes in the molecular-weight-distribution of the gelatin solution were less likely to affect the size-distribution of the nanoparticles. The temperature, $37^{\circ} \mathrm{C}$, was also selected to ensure that the molecular-weightdistribution remained relatively unaltered during incubation. The selected $\mathrm{pH}$ had to be such that the gelatin molecules would be sufficiently uncharged to remain sensitive to desolvation but sufficiently

Table 4. Mean size (nm) and polydispersity of gelatin nanoparticles (photon correlation spectroscopy) prepared by the optimized process.

\begin{tabular}{|c|c|c|c|c|c|c|c|}
\hline \multirow[b]{2}{*}{$\mathrm{pH}$} & & \multicolumn{6}{|c|}{ Ethanol concentration $(\mathrm{w} / \mathrm{w})$} \\
\hline & & $62 \cdot 5$ & 65 & $67 \cdot 5$ & 70 & $72 \cdot 5$ & 75 \\
\hline \multirow[t]{2}{*}{$5 \cdot 5$} & Size & I.S. & I.S. & I.S. & I.S. & I.S. & I.S. \\
\hline & Polydispersity & - & - & - & - & - & - \\
\hline \multirow[t]{2}{*}{$6 \cdot 0$} & Size & I.S. & $240 \cdot 7 \pm 24 \cdot 4$ & $228 \cdot 5 \pm 10 \cdot 2$ & $199 \cdot 3 \pm 13 \cdot 6$ & $232 \cdot 2 \pm 25 \cdot 1$ & I.S. \\
\hline & Polydispersity & - & $0.130 \pm 0.077$ & $0 \cdot 141 \pm 0.078$ & $0.115 \pm 0 \cdot 103$ & $0 \cdot 112 \pm 0.087$ & - \\
\hline \multirow{2}{*}{$6 \cdot 5$} & Size & I.S. & $210 \cdot 4 \pm 10 \cdot 2$ & $219 \cdot 1 \pm 12 \cdot 5$ & $205 \cdot 8 \pm 6 \cdot 8$ & I.S. & I.S. \\
\hline & Polydispersity & - & $0.094 \pm 0.049$ & $0.097 \pm 0.100$ & $0.119 \pm 0.076$ & - & - \\
\hline \multirow[t]{2}{*}{$7 \cdot 0$} & Size & I.S. & $189.9 \pm 7.8$ & $197.8 \pm 11.1$ & $192 \cdot 3 \pm 13.8$ & $221 \cdot 5 \pm 20 \cdot 2$ & I.S. \\
\hline & Polydispersity & - & $0 \cdot 115 \pm 0 \cdot 040$ & $0.107 \pm 0.059$ & $0.109 \pm 0.117$ & $0.135 \pm 0.083$ & - \\
\hline \multirow[t]{2}{*}{$7 \cdot 5$} & Size & I.S. & $224 \cdot 9 \pm 11 \cdot 4$ & $180 \cdot 8 \pm 12 \cdot 5$ & $179 \cdot 3 \pm 8 \cdot 6$ & I.S. & I.S. \\
\hline & Polydispersity & - & $0.080 \pm 0.045$ & $0.067 \pm 0.050$ & $0.097 \pm 0.066$ & - & - \\
\hline \multirow{2}{*}{$8 \cdot 0$} & Size & I.S. & $193 \cdot 3 \pm 11 \cdot 2$ & $177 \cdot 3 \pm 11 \cdot 2$ & I.S. & I.S. & I.S. \\
\hline & Polydispersity & - & $0.089 \pm 0.063$ & $0.133 \pm 0.071$ & - & - & - \\
\hline \multirow{2}{*}{8.5} & Size & I.S. & I.S. & I.S. & I.S. & I.S. & I.S. \\
\hline & Polydispersity & - & - & - & - & - & - \\
\hline
\end{tabular}

Values are means \pm s.e.m. of six readings. I.S. $=$ Insufficient scattering. 
charged to prevent their aggregation during nanoparticle formation. As noted, the IEP of B-type gelatins was in the range $4 \cdot 8-5 \cdot 2$ and $\mathrm{pH} 7$ was selected.

This optimized methodology produced dispersions of gelatin nanoparticles with mean sizes of 200 to $250 \mathrm{~nm}$ and polydispersity less than $0 \cdot 2$, as measured by light-scattering. Electron microscopy revealed that the preparations consisted of dispersions of individual primary gelatin nanoparticles, as opposed to other multiparticulate aggregates obtained using the method described by Marty et al (1978). It was shown by a non-parametric one-way analysis of variance test that the mean size and mean polydispersity of the gelatin nanoparticle populations obtained under these conditions (Table 4) were statistically identical $(\mathrm{H}=15.00$ at df 15 , $P>0.05)$. At $\mathrm{pH}<6.0$ the lack of net charge on the gelatin particles resulted in increased aggregation. At $\mathrm{pH}>7.5$ the increased charge on the particles resulted in increased resistance to dehydration, as shown by a lack of increase in scattering intensity of the gelatin solutions after addition of ethanol. The gelatin particles were also found to be stable to freezing and lyophilization. Three particle preparations with a mean size ( \pm s.e.m.) of 232.4 $( \pm 2.1) \mathrm{nm}$ and a mean polydispersity of 0.067 $( \pm 0.036)$ were lyophilized, stored for 2 weeks at room temperature and reconstituted with water. The reconstituted preparations had a mean size of $234.9( \pm 3.8) \mathrm{nm}$ and a mean polydispersity 0.133 $( \pm 0.039)$, both statistically identical (unpaired $t$ test, $t=0.576$ and 1.244 at df $10, P<0.05)$ ).

This optimized methodology for the production of gelatin nanoparticles has a number of advantages over previously published techniques (e.g. that of Marty et al 1978). The method produces dispersions of individual primary gelatin nanoparticles rather than multiparticulate aggregates (Figure 4). The method was also sufficiently robust that small changes of $\mathrm{pH}$ and ethanol concentration did not materially affect the size of nanoparticles produced. Thus, by systematic characterization of the time-dependent effect of temperature, $\mathrm{pH}$ and desolvating agent on the molecular-weight profiles, a process for precipitation and separation of dispersions of primary gelatin nanoparticles could be optimized.

\section{References}

Cantor, C. R., Schimmel, P. R. (1980) The kinetics of ligand interactions. In: Cantor, C. R., Schimmel, P. R. (eds) Biophysical Chemistry, Part III, The Behavior of Biological Macromolecules, Freeman and Company, San Francisco, pp $887-938$
Courts, A. (1954) The $N$-terminal amino acid residues of gelatin 2. Thermal degradation. Biochem. J. 58: 74-79

El-Samaligy, M., Rohdewald, P. (1982) Triamcinolone diacetate nanoparticles, a sustained-release drug-delivery system suitable for parenteral administration. Pharm. Acta Helv. 57: 201-204

El-Samaligy, M., Rohdewald, P. (1983) Reconstituted collagen nanoparticles. A novel drug-delivery system. J. Pharm. Pharmacol. 35: 537-539

Elysée-Collen, B., Lencki, R. W. (1996) Protein ternary phase diagrams. 1. Effect of ethanol, ammonium sulfate, and temperature on the phase behavior of type B gelatin. J. Agric. Food Chem. 44: 1651-1657

Flory, P. J., Weaver, E. S. (1960) Helix $\leftrightarrow$ Coil transitions in dilute aqueous collagen solutions. J. Am. Chem. Soc. 82: 4518-4525

Johns, P., Courts, A. (1977) Relationship between collagen and gelatin. In: Ward, A. G., Courts, A. (eds) The Science and Technology of Gelatin, Academic Press, New York, pp $138-178$

Jones, R. T. (1987) Gelatin: physical and chemical properties. In: Ridgway, K. (ed.) Hard Capsules: Development and Technology, The Pharmaceutical Press, London, pp $31-48$

Kreuter, J. (1994) Nanoparticles. In: Kreuter, J. (ed.) Colloidal Drug-Delivery Systems, Marcel Dekker, New York, pp 219-342

Lorry, D., Vedrines, M. (1985) Determination of molecularweight-distribution of gelatines by H.P.S.E.C. In: AmmannBrass, H., Pouradier, J. (eds) Photographic Gelatin. Proc. Fourth IAG Conf., Fribourg, 1983, International Working Group for Photographic Gelatin, Fribourg, pp 35-54

Marty, J. J., Oppenheim, R. C., Speiser, P. (1978) Nanoparticles - a new colloidal drug-delivery system. Pharm. Acta Helv. 53: 17-23

Oppenheim, R. C. (1986) Nanoparticulate drug delivery systems based on gelatin and albumin. In: Guiot, P., Couvreur, P. (eds) Polymeric Nanoparticles and Microspheres, CRC Press, Florida, pp 1-26

Perrin, D. D., Dempsey, B. (1974) Composition-pH tables of some commonly used buffers. In: Perrin, D. D., Dempsey, B. (eds) Buffers for $\mathrm{pH}$ and Metal-Ion Control, Chapman and Hall, London, pp 128-156

Piez, K. A., Weiss, E., Lewis, M. S. (1960) The separation and characterization of the $\alpha$ - and $\beta$-components of calf-skin collagen. J. Biol. Chem. 235: 1987-1991

Piez, K. A., Lewis, M. S., Martin, G. R., Gross, J. (1961) Subunits of the collagen molecule. Biochim. Biophys. Acta 53: 596-598

Privalov, P. L., Gill, S. J. (1988) Stability of protein structure and hydrophobic interactions. Adv. Protein Chem. 39: 191234

Steckert, J., Kramer, D., Brand, J. (1992) Preparative chromatography and analysis of molecular-weight fractions of gelatin. J. Photogr. Sci. 40: 134-137

Steven, F. S., Tristram, G. R. (1962) The denaturation of acetic acid-soluble calf-skin collagen. Biochem. J. 85: $207-210$

Veis, A., Anesey, J., Cohen, J. (1962) The characterization of the $\theta$-component of gelatin. Arch. Biochem. Biophys. 98: $104-110$

Von Hippel, P. H., Harrington, W. F. (1959) Enzymic studies of the gelatin collagen-fold transition. Biochim. Biophys. Acta 36: $427-447$ 